\title{
Modelación de la educación y escolaridad desde las revoluciones industriales
}

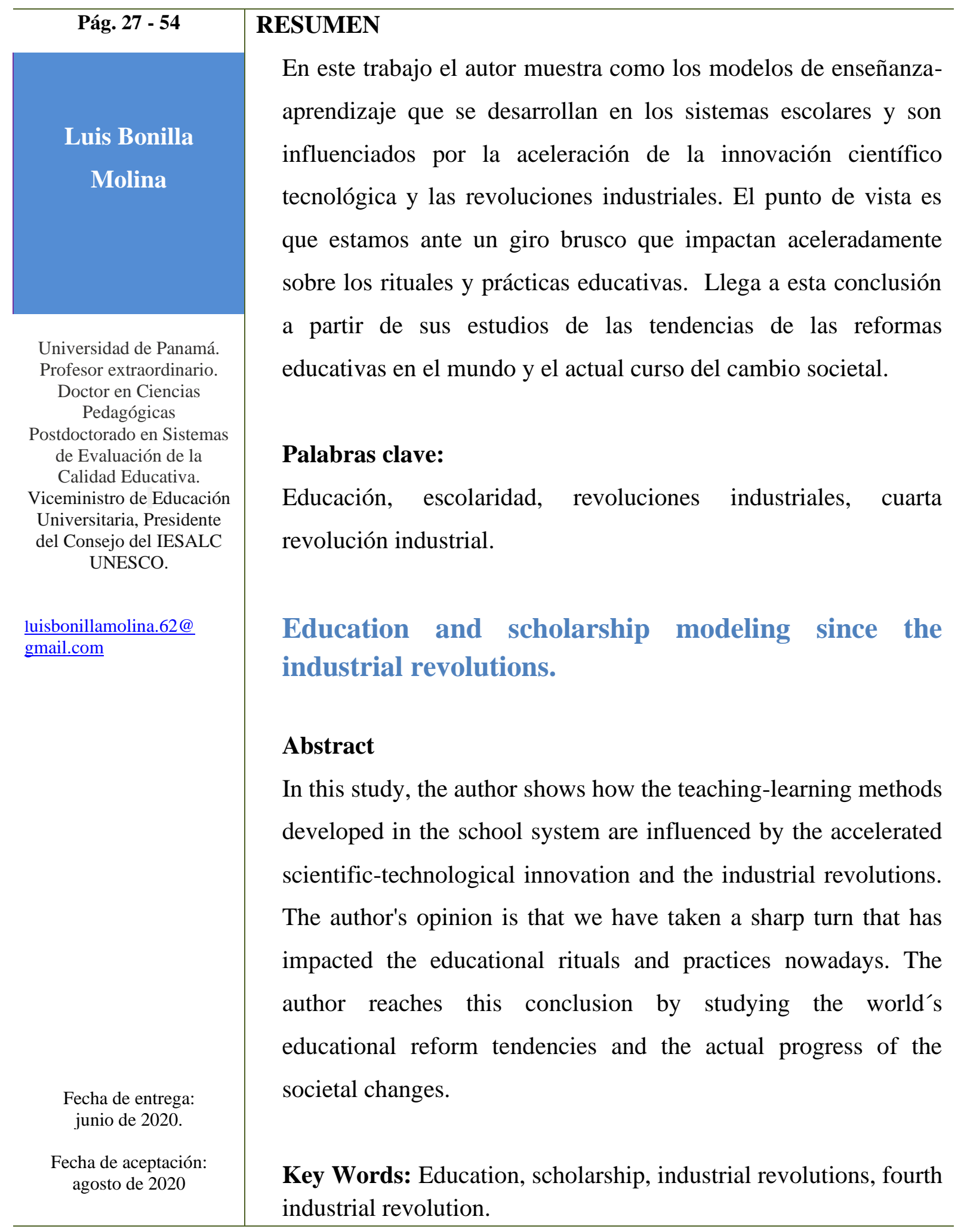




\section{Perspectiva para entender lo que ocurre}

Hay una abundante bibliografía que procura explicar las causas y sentido de eso que se ha denominado como "crisis educativa". He insistido que la definición de "crisis" es una construcción que ha usado el neoliberalismo para justificar la vorágine de reformas y contrarreformas educativas que se han impuesto desde la década de los ochenta del siglo pasado con el fin de destruir la escuela pública. Además, la noción de crisis de lo educativo intenta desviar la atención a lo escolar y educativo como si ello fuera una isla en sí misma, desconectada de las dinámicas sociales, económicas, políticas, culturales y tecnológicas. En otros casos lo educativo/escolar y las "crisis educativas" se presentan como temas nacionales, desconectados del sistema mundo capitalista y sus dinámicas.

Cuando se supera el temor a ser acusado de economicistas, muchos analistas le entran a lo económico, pero muchas veces se atascan en la dimensión meta ideológica del capital y no se adentran en las relaciones específicas del modo de producción con lo educativo. Ello adquiere dimensiones preocupantes cuando evidenciamos la desconexión existente en muchos análisis educativos, respecto a la relación de lo "pedagógico" con la aceleración de la innovación, su impacto en el modo de producción y acumulación, respecto a las dinámicas de enseñanzaaprendizaje.

Por ello, la perspectiva asumida en el presente trabajo es valorar de manera especial el impacto de aceleración de la innovación científica-tecnológica en el modo de producción y acumulación capitalista, así como en las demandas que ello genera sobre lo educativo. Por supuesto que esta no es una perspectiva neutra, tiene la objetividad de la lucha de clases y las resistencias anticapitalistas.

En consecuencia, consideramos de especial significación recuperar la tradición de estudios sobre la relación de la escuela con la fábrica, pero entendiendo que la propia fábrica está cambiando, está mutando. Las fábricas 4.0 comportan una redefinición de parámetros y exigencias sobre la escuela que no pueden ser comprendidos desde la lógica de la máquina educativa newtoniana. 
Por ello, muchos de los análisis de lo educativo/escolar que descuidan esta realidad, corren el riesgo de hacer teoría mirando al retrovisor.

Desde la izquierda y las pedagogías críticas siempre tuvimos claridad sobre el carácter reproductor del sistema, asignado por el capitalismo a la escuela. Unos la vieron simplemente como un aparato ideológico del Estado, otros la entendimos como ello, pero también como un lugar de disputa, de resistencias, porque estaba constituida por seres humanos, en su inmensa mayoría explotados.

Defendimos a la escuela en su dimensión democratizadora del conocimiento que había sido apropiado durante siglos por los instalados en el poder. Defendimos la escuela porque abría la posibilidad que millones de trabajadores y sus familias comprendieran de manera científica al mundo, derrotando el oscurantismo de los dogmas religiosos. Defendimos la escuela porque era un espacio de encuentro, para la construcción de tejido social, para la formulación compartida de resistencias anticapitalistas.

Estábamos conscientes, en mayor o menor medida, que la escuela para el capitalismo no solo intentaba reproducir el performance de la fábrica con la carga de explotación, fragmentación y apropiación de la plusvalía, en ese caso ideológica, sino que también era otra máquina de control social. A esa máquina newtoniana educativa decidimos intentar aflojarle las tuercas, para desarmarla andando, construyendo en el proceso saber pedagógico emancipador, prácticas educativas liberadoras. Unas veces avanzamos, otras nos atascamos y en algunos casos incluso ajustamos la máquina de dominación con palabras radicales que se convertían en significantes vacíos, narrativas funcionales a la propia dominación.

Defendimos la escuela pública por sus posibilidades para construir educación popular, crítica, científica y alternativa. Hicimos de la disputa escolar una posibilidad para pensar otra escuela, en otro tipo de sociedad, que para nosotros es la socialista democrática. No fue nuestra intención defender a la máquina educativa newtoniana, lo que ocurre es que entramos en caos cuando esa máquina se convirtió en obsoleta para el capitalismo y comenzó a transformarse en una nueva máquina. Con el emerger de la tercera revolución industrial la escuela capitalista comenzó a mutar y la incomprensión estructural del cambio en curso, generó en algunos casos un 
pensamiento conservador de la izquierda pedagógica, que nos llevó a abrazarnos al viejo modelo como si fuera un salvavidas ante nuestra incapacidad de pensar lo alternativo en lo nuevo.

Y estábamos en desconcierto paradigmático funcional cuando se nos comenzó a anunciar y mostrar la nueva máquina educativa propio del capitalismo de la cuarta revolución industrial. Intentemos en consecuencia analizar la escuela y los sistemas escolares en relación con las revoluciones industriales y la aceleración de la innovación.

\section{Primeras revoluciones industriales y educación}

El capitalismo fabril significó un reordenamiento extraordinario de la economía, la cultura, las sociedades y la incorporación de este modelo de desarrollo al plano del currículo educativo. En la larga transición del feudalismo al capitalismo la escuela adquirió un nuevo rostro, una nueva conceptualización. La ilustración y el desarrollo científico de los siglos XVIII y XIX permitieron el emerger de la primera y segunda revolución industrial, con impacto directo en la conformación e impulso del capitalismo industrial. El aprendizaje basado en la memoria ya no resultaba suficiente, se requería memoria + comprensión de su génesis y líneas de expansión, para seguir ampliando y especializando el conocimiento que requería en ese momento el capital y que demandaba la aceleración de la innovación tecnológica.

Este capitalismo fabril estructuró los saberes conforme a su utilidad para mejorar, optimizar y potenciar, las máquinas sobre las cuales se estructuraba la producción y la sociedad. El capitalismo fortaleció la división del conocimiento en campos (Ciencias naturales, ciencias sociales, ciencias humanísticas, ciencias duras, filosofía) y estos a su vez en disciplinas. Los principios que orientaron esta estructuración fueron los prolegómenos de lo que hoy conocemos como la eficiencia y la eficacia del conocimiento. El capitalismo estructuró el mundo y sus instituciones a la imagen y semejanza de las máquinas que surgieron en la primera y segunda revolución industrial. 
La lógica de la máquina newtoniana impregnó a la implementación de las pedagogías y la escuela, liceo y universidad. Mientras la enseñanza se fundamentó en la pedagogía vista como componentes ensamblables (didáctica, currículo, evaluación, planeación, gestión), el aprendizaje fue valorado como productos que se replicaban y evaluaban conforme a la lógica de producción de las mercancías generadas en las fábricas-escuelas. Los sistemas escolares se estructuraron a partir de las premisas de Comenio (maternal=preescolar, común=primaria, gimnasio=bachillerato y academia=universidad) y como segmentos de producción separados, pero ensamblables, estructurados según su complejidad (edad, desarrollo físico). La premisa de Comenio que señalaba que la educabilidad estaba en la naturaleza de los seres humanos se convirtió en el correaje de la lógica capitalista sobre la escuela.

La perspectiva de Comenio se fue enriqueciendo por otras propuestas, las cuales han trabajado en otros momentos. El conocimiento requería entonces memoria, información, comprensión y manejo contextual de procesos mecánicos, abriendo la posibilidad para la experimentación y los desarrollos conceptuales que eran valorados bajo el prisma de la utilidad para el sistema, es decir para algunas de las máquinas del capitalismo económico, político, social, cultural y tecnológico.

La idea que para mejorar cada todo (máquina) había que perfeccionar las partes (ensamblables) y evolucionar al detalle los mecanismos (sistemas), lo que facilitó la construcción de una epistemología disciplinar de la educación, de los procesos de enseñanza-aprendizaje en los sistemas escolares.

Las dinámicas de las escuelas se estructuraron conforme a la mirada de la organización escolar de Comenio y la lógica disciplinar reforzó la idea de los nodos como materias. Las materias se dividían en objetivos secuenciales, a la usanza de una cadena de producción y, las evaluaciones cumplían la función de control en la producción de conocimientos (mercancías). La correlación de objetivos surgió como la expresión de especialización con complementariedad con otros conocimientos. 
La formación docente se especializó al interior de los componentes de las pedagogías, surgiendo especialistas en cada una de las partes, a tal punto que al final apenas si conocían el todo; la idea era que, a los directores, supervisores y autoridades educativas en general, les correspondería coordinar el ensamblaje de los procesos.

Surgieron los especialistas en currículo, evaluación, planeación, didácticas, innovaciones, gestión del aula, gestión de plantel. El todo se fragmentó en las partes y la pedagogía dejó de comprenderse en su relación con el todo social. Pero esto era lo que necesitaba el capitalismo de la primera y segunda revolución industrial. En los preludios de la tercera revolución industrial, la especialización en pedagogía derivaría en autonomía de las partes de la máquina pedagógica (currículum versus didácticas, evaluación versus gestión, etc.), sin que llegaran a ser nuevas máquinas, sino prototipos que competían entre sí.

\section{Tercera revolución industrial y procesos de enseñanza aprendizaje}

Las máquinas de la tercera revolución industrial (1961-2021/2025), no eran ya de ensamblaje y fricción, de movimiento, aceleración y resistencia, sino que funcionaban con circuitos e interconectividad, con impulsos y pulsos binarios. Las máquinas de este periodo se construían fusionando principios que antes habían sido estancos separados por las disciplinas. Los tornillos, poleas, engranajes eran cada vez menos, los rasgos de las máquinas del capitalismo de la tercera revolución industrial. Las máquinas sociales, culturales, económicas, políticas y tecnológicas que habían sido construidas bajo la lógica de la primera y segunda revolución industrial comenzaron a ver saltar por los aires sus tuercas (narrativas, imaginarios), ruedas (prácticas, protocolos) y ligamentos (instituciones).

La epistemología del capitalismo de la primera y segunda revolución industrial entró en una fase de mutación en la tercera revolución industrial, sin embargo, una parte importante de las resistencias anticapitalistas no pudieron ver esta "evolution" del capital y continuaron haciendo política para enfrentar a la vieja máquina; algo similar ocurrió con la izquierda pedagógica y un segmento significativo de las pedagogías críticas. 
La tercera revolución industrial con el emerger de la lógica computacional construyó la sensación de caos en la enseñanza, el aprendizaje y el conocimiento. Para seguir avanzando en el desarrollo de las nuevas máquinas y del capitalismo en su conjunto, los estancos disciplinares resultaban obsoletos para el propio capital. Ya la máquina educativa newtoniana resultaba insuficiente para lo nuevo, orientado por los principios de la lógica binaria y la física cuántica.

Jóvenes, niños y niñas percibían mejor esa relación asincrónica, entre una máquina escolar newtoniana que se estaba desbaratando y nuevos funcionamientos de la máquina educativa capitalista de la tercera revolución industrial que comenzaba a mostrarse. Los adultos, los y las profes, educados en los centros de formación docente para enseñar y ver el aprendizaje conforme a la vieja máquina educativa, en muchos casos solo alcanzábamos a ver la posición de los más chicos como déficit de atención, problemas de sociabilidad y otras seudo denominaciones, cuando en realidad lo que estaba ocurriendo era que ellos estaban captando mucho mejor nuevos ritmos y características de la máquina educativa social, política, económica, cultural y tecnológica del capitalismo del siglo XXI; a estos chicos les parecía (y parece), que cuando iban a la escuela estaban entrando a un museo.

La responsabilidad no era de los y las maestras(os), sino del centro capitalista que no terminaba de entender cómo podía formalizar, poner en marcha y controlar a las nuevas máquinas sociales que estaban surgiendo. Por ello, el periodo de los sesenta y parte de los setenta del siglo XX fue de inestabilidad, que se resuelve con apelaciones al autoritarismo o al dejar hacer. En educación esta inestabilidad se encubrió con la falsa etiqueta de "crisis educativa".

Lo que si teníamos claros desde las pedagogías críticas era que había que transformar la escuela (aunque no terminábamos de saber cómo) para defender el derecho a la educación de todos y todas, especialmente de los más pobres y excluidos que estaban marginados del acceso a lo nuevo, a los "juguetes" y "artefactos" del mundo tecnológico de la tercera revolución industrial. La escuela, la escuela pública, era y es, donde se puede generar el encuentro humano más allá de la fragmentación y dominación de la sociedad de clases, para analizar, comprender, valorar y decidir sobre esa nueva vorágine tecnológica que no terminaba de llegar a casa, pero que está determinando al ser social. 
Pero voluntad requiere de conocimiento científico y a decir verdad faltaron espacios y flexibilidad conceptual, paradigmática, para entender la complejidad de lo nuevo. Pero la incomprensión por parte de las resistencias anticapitalistas al respecto no detenía la lucha de clases, ni dejaba de hacer sentir las nuevas formas de opresión.

Los campos experimentales de conocimientos vinculados al capitalismo de las décadas del setenta, ochenta y noventa del siglo XX, comenzaron a conformar equipos multidisciplinarios que abrieron paso al paradigma transdisciplinario. El problema fue que las máquinasescuelas, máquinas-liceos, máquinas-universidades, nunca supieron por dónde comenzar a desandar el cambio. No entendían cuáles piezas había que cambiar, que elementos había que fusionar, que tenía que desaparecer y que había que crear, porque la auto imagen institucional sostenida por décadas era la de una máquina educativa newtoniana. Esto fue lo que se encubrió con la etiqueta de "crisis educativa".

Una morisqueta de este "debate-acción” hizo que, desde las disciplinas y las materias, se apelara al cliché de lo transdisciplinario, sin terminar de construir una máquina escolar transdisciplinaria. Pero la parálisis, el inmovilismo, no es el rasgo de la dominación, ni de la lucha de clases.

Fuimos tan "ingenuos" en la izquierda pedagógica que pretendimos apropiarnos de la transdisciplinariedad como propia, decir que ser transdisciplinario era una forma de resistir a la visión disciplinar del capitalismo, cuando en realidad nos estábamos refiriendo al capitalismo de la primera y segunda revolución industrial. No terminamos de entender que la transdisciplinariedad era ahora la mirada del capitalismo de la tercera revolución industrial, la perspectiva era construir, la ontología de las nuevas máquinas. Entonces, ya no estábamos resistiendo con la simple apelación a la transdisciplinariedad porque el capitalismo había asimilado esta premisa para mejorar su funcionamiento estructural.

Las reformas educativas solo podían intentar limar superficialmente o pulir partes de la vieja máquina educativa newtoniana, pero eso sí, servían para destruir el concepto público de la educación, para "poner en evidencia" a los docentes y destruir la profesión docente, para abrir paso a la desinversión en materia educativa. Por ello, las reformas se convertían en lo táctico en 
contrarreformas que paleaban y hacían control de daños, mientras lo sustantivo de la transformación radical de los procesos de enseñanza-aprendizaje se colocaba en la externalidad de los sistemas escolares.

Desde la lógica del capital dos elementos fueron moldeando la ruta para construir hegemonía respecto a las supuestas dimensiones de la "crisis de los sistemas educativos", la profesión docente y la escuela. Se procuraba instalar la idea en la ciudadanía que "algo andaba mal en lo educativo", para crear las condiciones de posibilidad de diseño de otra escuela capitalista, abriéndole paso a los conceptos necesario para comprender y poner en funcionamiento a la nueva máquina educativa del capitalismo cognitivo de la tercera revolución industrial.

El primero, la construcción de cultura evaluativa y, el segundo, debates e iniciativas desde la externalidad escolar, desde los bordes institucionales para poner en evidencia el impacto del mundo digital en los procesos de enseñanza-aprendizaje y la precaria capacidad de la escuela pública, los centros de formación docente y los y las maestras(os) para adaptarse a los nuevo. Veamos cada uno de ellos.

\section{Cultura evaluativa}

En el denominado Informe Faure (Faure y Otros, 1973, p. 58), señala que: "La revolución industrial, a medida que alcanza un número mayor de países, exige una expansión de la educación y lleva consigo la aparición del concepto de instrucción universal y obligatoria, históricamente unido al de sufragio universal".

Se precisó en este Informe aludido (p. 60), que la educación es "en términos presupuestarios ocupa el segundo de los gastos públicos mundiales, inmediatamente después de los gastos militares" a lo cual agregan, que a pesar de esto: 
La sociedad rechaza los productos de la educación... [ya que] mientras hasta el presente las sociedades en lenta evolución (excepto cortas fases de mutación) absorbían fácilmente y de buena gana los productos de la educación, o por lo menos se acomodaban a ellos, en la actualidad no ocurre otro tanto (p. 62).

Esta perspectiva del Informe Faure (Faure y Otros, 1973) induce a pensar la necesidad de comprobar la eficiencia de los costos educativos respecto a sus resultados, elemento que constituiría más adelante el lugar de enunciación de la Cultura evaluativa. Señala el mencionado Informe, que se evidencian dos elementos causales del hecho que "la sociedad rechaza los productos de la educación”. Estos elementos, continúa el Informe Faure, muestran que la educación, en la tercera revolución industrial, no cumplía con dos aspectos que habían sido referentes básicos de su accionar a través del tiempo, en sus puntos: “a) la educación precede y, b) la educación prevé” (Faure y Otros, 1973, pp. 61-62).

De hecho, la educación había precedido el "desarrollo económico", sobre las sociedades a partir de la revolución industrial (preciso de la primera y segunda revolución industrial), pero esta dinámica comienza a romperse con el desarrollo de la tercera revolución industrial, algo que el propio Informe Faure no logra visualizar en toda su complejidad.

Por otra parte, señala dicho Informe Faure en el punto b) La educación prevé que "por primera vez en la historia la educación se emplea conscientemente en preparar a los hombres para tipos de sociedades que todavía no existen” (p. 62), lo cual constituye una campanada de alerta respecto al desfase que se comienza a evidenciar entre el ayer y el hoy, entre el despegue de la tercera revolución industrial y lo que se enseña en escuelas, liceos y universidades propio de la primera y segunda revolución industrial.

Los "tres fenómenos nuevos" enunciados en el Informe Faure (precede y prevé que la sociedad rechaza los productos de la educación) colocan en evidencia la inconsistencia de la relación entre aceleración de la innovación científico-tecnológica y educación en el marco del capitalismo de la tercera revolución industrial. 
El desembarco de la globalización económica y la nueva mundialización cultural de la tercera revolución industrial incorporan con fuerza al léxico educativo la noción de exclusión como complemento a la idea de cobertura escolar. La idea de exclusión jugaría un rol central en las justificaciones para la instauración de la cultura evaluativa, en el marco de la reestructuración capitalista, uno de cuyos elementos dinamizadores era (y es) la aceleración de la innovación.

La reestructuración capitalista derivada del impacto de la tercera revolución industrial, la internacionalización del capital, es decir de la globalización, impulsan la crítica a los Estados Nacionales, su tamaño y el precario cumplimiento de las metas que se plantea el capital para las economías y sociedades nacionales. Este cuestionamiento culmina en los ochenta del siglo XX en propuestas de Reforma de los Estados Nacionales que se justifican por las llamadas crisis de eficacia y legitimidad de la acción pública, entre ellas la Comisión Económica para América Latina y el Caribe (CEPAL), Instituto Latinoamericano y del Caribe de Planificación Económica y Social (ILPES) dependencia de la CEPAL, Banco Interamericano de Desarrollo (BID), Banco Mundial, entre otros.

Argumentan las llamadas bancas de desarrollo y los organismos multilaterales del sistema de Naciones Unidas que la crisis es de eficacia porque las instituciones públicas no sirven para lo que están diseñadas y puestas en marcha y, de legitimidad, porque no expresan los intereses de los ciudadanos. En este último caso, la preocupación no es por la gente, sino porque las instituciones no sirven para crear el nuevo paradigma de disminución del gasto público y que cada uno comience a gestionar su vida; la legitimidad burguesa se vincula a la intención que los ciudadanos asuman una parte importante de los costos de la agenda social.

Estos señalamientos tienen un correlato en lo educativo, al indicar que la crisis de eficacia del Estado se expresa en crisis de calidad educativa (eficacia=calidad) y, la crisis de legitimidad en precaria pertinencia de la acción escolar (legitimidad=pertinencia educativa). La "crisis educativa" adquiere categorías fuerza que orientan el discurso neoliberal. 
La calidad educativa pasa a ser un comodín que sirve para justificar cualquier crítica a la educación, mientras que la pertinencia profundiza el consenso social respecto a lo señalado por el Informe Faure (UNESCO, 1973, p. 62) y que en el punto c) se explicita que la sociedad rechaza los productos de la educación.

Para resolver los problemas de calidad y pertinencia educativa, el Banco Mundial (BM), la Organización para la Cooperación y el Desarrollo Económicos (OCDE), el Banco Interamericano de Desarrollo (BID), el Banco de Desarrollo de América Latina (CAF) y la propia UNESCO comenzaron a señalar con fuerza, en los ochenta del siglo $\mathrm{XX}$, que era necesario evaluar los sistemas educativos y los aprendizajes. Mientras tanto, los centros capitalistas siguen sin definir claramente como quieren que funcionen y trabajen escuelas, liceos y universidades, eso sí propagan la idea que la educación gestionada por privados es mejor que la pública.

La globalización y la mundialización cultural demandaban la homogenización de la sociedad mundial y la estandarización de las políticas públicas. La cultura evaluativa se convierte en una herramienta fundamental para esos fines. En ese contexto de 1990 la UNESCO decide convocar a la Conferencia Mundial sobre Educación para Todos: Satisfacción de las Necesidades Básicas de Aprendizaje, la cual se realizó en Jomtien (UNESCO-Jomtien, 1990).

La Declaración Mundial sobre Educación para Todos, "Satisfacción de las necesidades básicas de aprendizaje” que emana de esta Conferencia (UNESCO-Jomtien, 1990, párr. 8) aprobada por 155 países, concluye en una parte del Preámbulo que:

El mudo está en vísperas de un nuevo siglo, cargado de promesas y de posibilidades. Hoy somos testigos de un auténtico progreso hacia la distensión pacífica y de una mayor cooperación entre las naciones. Los derechos y las aptitudes fundamentales de las mujeres se están convirtiendo en realidad. El volumen mismo de información existente en el mundo, mucho de ello útil para la supervivencia del hombre y para su bienestar elemental, es inmensamente mayor que el disponibles hace solo pocos años y su ritmo de crecimiento continúa acelerándose. 
La Declaración de Jomtein coloca su preocupación sobre el impacto en lo educativo de la innovación y la aceleración de los descubrimientos. Educación para Todos (EPT) se plantea trabajar a nivel gubernamental y con la sociedad civil para evitar que se amplié el rezago de millones de niños, niñas y adolescentes y para mejorar los indicadores de cobertura de la educación básica a nivel planetario.

El paradigma de EPT es que si quedan niños, niñas y adolescentes fuera de la escuela no podrán comprender lo nuevo que está surgiendo, ni insertarse en el mundo, ampliando con ello la brecha de exclusión; la educación sigue jugando de esta perspectiva un papel igualador. Sin embargo, también EPT surge como el enunciado que reúne el esfuerzo de estandarización y homogenización de las políticas educativas de la educación básica para procurar romper las desigualdades y exclusiones.

A mediados de la década de los noventa la UNESCO conforma una Comisión para elaborar el Informe Internacional sobre Educación para el Siglo XXI, constituida por Jacques Delors (presidente), Al Mufti, Isao Amagi, Roberto Carneiro, Fay Chung, Bronislaw Geremek, William Gorham, Aleksandra Kornhauser, Michael Manley, Marisela Padrón Quero, Marie-Angélique Savané, Karan Singh, Rodolfo Stavenhagen, Myong Won Suhr, Zhou Nanzhao. Esta Comisión presenta el texto denominado "La educación encierra un Tesoro". El documento plantea un conjunto de temáticas, pero en este momento nos centraremos en destacar que el llamado Informe Delors (por el presidente de la Comisión) ratifica que "las opciones educativas son opciones de sociedad. Como tales, exigen en todos los países un amplio debate público, basado en la exacta evaluación de los sistemas educativos" (Delors y Otros, 1996, p. 38).

Delors y su equipo, avanzan en definir lo que consideran son los pilares fundamentales de la educación para y del siglo XXI: Aprender a conocer (lo nuevo), Aprender a Hacer (lo que ahora se necesita), Aprender a vivir juntos (en un nuevo modelo societal) y Aprender a vivir con los demás (los diversos, los rezagados), es decir en la pertinencia con equidad. Para Delors y Otros (1996), ello solo es posible alcanzarlo con cultura evaluativa en los sistemas escolares. 
El discurso e institucionalización de la cultura evaluativa marchan juntas y comienzan a constituirse en el centro de debates globales. Los elementos que acompañan esta operación política de reingeniería planetaria de los sistemas educativos son el discurso sobre la incapacidad de las escuelas, liceos y universidades para entender el impacto de la innovación, el cambio cultural de los más chicos y jóvenes, la onda transformadora del mundo digital en las aulas y las potencialidades de la educación virtual.

Una década después de Jomtein, en Dakar, abril de 2000, los países miembros se reúnen para monitorear EPT y trazar una estrategia de acompañamiento a los dos ODM (Objetivos de Desarrollo del Milenio) asociados a educación que construía Naciones Unidas. En el Foro Mundial sobre Educación (2000) llevado a cabo en Dakar se aprueba el llamado "Marco de Acción de Dakar: Educación para Todos: cumplir nuestros compromisos comunes (con los seis marcos de acción regionales)” (UNESCO, 2000, p. 8). También se menciona en dicho Marco de acción las dificultades y oportunidades descritas en el punto 15 al señalar que:

Los logros concretos, pero modestos, del último decenio imponen cierta reserva. En efecto, muchos países siguen teniendo dificultades para definir el sentido, la finalidad y el contenido de la educación básica en un mundo que cambia rápidamente, así como para evaluar los resultados y logros de la educación (p. 13).

En Dakar se reitera la mirada de Educación para Todos (EPT) de incluir en las aulas, igualar en la comprensión de lo nuevo y evaluar lo que se hace en educación. Mientras que la ONU impulsa los Objetivos de Desarrollo del Milenio (ODM, 2000-2015) como esfuerzo planetario de homologación de prácticas y decisiones gubernamentales, EPT se constituye en el espacio que desarrolla las premisas de los Informes de Faure y Delors (que veremos más adelante) en materia educativa. Ambas iniciativas, ODM y EPT son esfuerzos estandarizadores de políticas públicas.

En la Reunión de 2011 del Grupo de Alto Nivel sobre la EPT la Directora General de la UNESCO Dra. Irina Bokova pidió actuar con más apremio para lograr los objetivos de la Educación para Todos (UNESCO, 2011, párr. 4 )., afirmando que "la educación necesita, por 
último, enfoques innovadores para responder a las exigencias complejas de la mundialización y aprovechar plenamente las posibilidades ofrecidas por las tecnologías de la comunicación y la información” Por ello, la UNESCO sigue construyendo discurso para abrir paso a la cultura digital en el aula.

Durante el Periodo de impulso de la UNESCO con la EPT (1990-2015) mejoran sensiblemente los indicadores de cobertura y se consolida el paradigma de las competencias educativas, que no es otra cosa que tratar de adecuarse a lo que Faure consideraba como los fenómenos nuevos de la educación, la capacidad de preceder (al desarrollo económico y tecnológico) y de proveer (los profesionales requeridos).

Esta línea continuaría hasta el balance de cierre de la UNESCO-EPT (2015, p. 129) en Corea del Sur, con el documento que precisa a que se refiere cuando se habla de competencias, ya que "en muchos idiomas existen términos diferentes para referirse a ello, pero allí precisan que las capacidades son innatas y las competencias se adquieren mediante la experiencia en contextos tanto escolares como no escolares".

El recorrido previo a Incheon el discurso de las competencias se asocia a la rendición de cuentas, fortaleciendo la cultura evaluativa estandarizada, como lo expresa el texto de la Declaración de Lima (UNESCO, 2016a, p. 19), la que en su numeral diecinueve destaca la importancia de la Gobernanza y financiamiento, al afirmar que:

Estamos determinados a establecer marcos de política que promuevan la rendición de cuentas y la transparencia, y fortalezcan la participación de todos/as las y los actores involucrados (como las organizaciones de la sociedad civil, comunidades, familias, docentes / como, estudiantes y otros asociados locales) en todos los niveles del sistema educativo. Nos comprometemos a fortalecer la educación pública como garantía para la construcción de la democracia y el derecho a la educación. Los gobiernos permanecen como los garantes de derechos y custodios de la eficiencia, la equidad y la gestión y financiamiento sustentables para la educación y las escuelas. 
En Incheon Corea, durante el mes de mayo de 2015 se realizó el balance mundial final de Educación para Todos (EPT), con el propósito de revisar los logros desde Jomtein (1990), Dakar (2000), Jomtein (2011) hasta esa fecha y establecer metas futuras. La reunión de Incheon acuerda incorporar un objetivo educativo a los futuros objetivos de desarrollo de Naciones Unidas y su balance prospectivo se refleja en el documento titulado Educación 2030. Declaración de Incheon (UNESCO, 2016b).

En la Declaración de Incheon en el subtítulo Hacia 2030: una nueva visión de la educación (UNESCO, 2016b, p. IV), donde en el numeral 9 se plantea "Nos comprometemos con una educación de calidad y con la mejora de los resultados de aprendizaje, para lo cual es necesario fortalecer los insumos, los procesos y la evaluación de los resultados y los mecanismos para medir los progresos. (...)".

Es decir, el encuentro de EPT con los ODS se da en continuidad con la línea de trabajo emprendida en los setenta y ochenta del siglo XX, de evaluación justificada en la calidad, a lo que se complementan las nociones de inclusión y equidad.

Ese mismo año, en Nueva York, en septiembre de 2015, con la aprobación de 193 países surgen los diecisiete Objetivos de Desarrollo Sostenible (ODS) para el periodo 2015-2030. Los ODS son el consenso mundial para la orientación estratégica de las políticas públicas de los Estados nacionales, cuyo cumplimiento tiene impacto en los mecanismos de financiación internacional. Los ODS y en especial el ODS 4 parecieran estar en la línea de transferencia de la soberanía de lo nacional a lo supranacional, propia de las exigencias del modelo económico, político, social, cultural y tecnológico de la cuarta revolución industrial en proceso de desembarco. Apropiación de la soberanía nacional algo que ya había postulado el primer Director General de la UNESCO, el señor Julian Huxley como tarea del organismo multilateral.

Dentro de los ODS se contempla el número cuatro, denominado calidad educativa, que establece las metas e indicadores consensuados, en la nueva fase de estandarización mundial de políticas educativas, vinculada a la evaluación de la calidad. De hecho, dentro de la instrumentación del ODS 4 se contempla que durante el periodo de vigencia (2015-2030), todos los países deberán constituir organismos locales e independientes de evaluación de la calidad educativa. 
Tres décadas atrás, en el marco de la toma de control de los capitales nacionales sobre las economías y burguesías locales de América Latina y el Caribe (ALC), la Socialdemocracia impulsa en la década de los ochenta del siglo XX dos candidaturas abiertamente neoliberales: Carlos Salinas de Gortari en México y Carlos Andrés Pérez (CAP) en Venezuela. A CAP la meta le sería esquiva, mientras que Gortari lograba allanar el camino, Salinas de Gortari construye las condiciones de posibilidad para que la UNESCO, en 1994 bajo el mandato de su sucesor Ernesto Zedillo, en la ciudad de Monterrey, México, constituyera el Laboratorio Latinoamericano de Evaluación de la Calidad Educativa (LLECE).

El LLECE surge en 1994 con el auspicio y la bendición del neoliberalismo educativo. El LLECE-UNESCO escala la cultura evaluativa a otro nivel, dándole rango institucional, algo que desde los setenta se le venía planteando a los sistemas escolares en el mundo sin lograr mayores frutos. Plantea como ruta para evaluar los sistemas educativos, la valoración de los aprendizajes; así surgen las pruebas PERCE (LLECE-UNESCO, 1997), SERCE (LLECE-UNESCO, 2004/2008), TERCE ((LLECE-UNESCO, 2015), y actualmente el ERCE (LLECE-UNESCO, 2019), en dieciocho países de América Latina y el Caribe (ALC). El LLECE le reporta a la dirección de estadísticas de la UNESCO, uno de los despachos en los cuales gravitaría como funcionario de este órgano multilateral el señor Andreas Schleicher.

En 1995, Schleicher quien para ese entonces formaba parte del equipo de Educación de la UNESCO, le plantea en una reunión en París a los representantes 28 países pertenecientes a la OCDE, la posibilidad de realizar "un examen global que permita comparar a nivel internacional el rendimiento de cada sistema educativo y establecer herramientas para su mejora" (El Confidencial, 2018), sustentado en el hecho que la conformación del LLECE no había generado resistencias en gremios, sindicatos, intelectualidad ni en los gobiernos; es decir la hegemonía estaba madura para comenzar a dar frutos prácticos a escala planetaria.

Schleicher no logró conseguir apoyos inmediatos, pero tejió un vínculo nunca explicado entre la UNESCO y la OCDE, para el montaje de las pruebas PISA. En 2001 se conocería los resultados iniciales de la primera generación de pruebas PISA (2001-2007) y posteriormente, el otrora 
funcionario de UNESCO, Andreas Schleicher comenzaría a dirigir personalmente (ya no a control remoto desde UNESCO), la segunda generación de las pruebas PISA (2008-2020).

La cultura evaluativa contaba ahora con instituciones, una regional el LLECE y otra mundial la OCDE-PISA, quienes bajo el pretexto de monitorear los aprendizajes y la calidad educativa refuerzan lo que Faure definía como "la sociedad rechaza los productos de la educación". Estas instituciones tratan de construir la nueva máquina educativa de la tercera revolución industrial, a partir del desmontaje de la anterior, con las herramientas que provee la evaluación estandarizada.

En 2014 la UNESCO convoca a una Comisión Internacional que actualice los Informes FaureUNESCO (1973) y Delors (1996), planteando los desafíos actuales de la Educación. Esta Comisión presentaría el documento Replantear la Educación: Hacia un bien común mundial (UNESCO, 2015a). Este Informe implica un salto en la conceptualización de la estandarización de políticas educativas, la cultura evaluativa y gobernanza educativa supranacional y, que colocaría en relieve el "desfase cada vez mayor entre educación y empleo", el papel de los "educadores en la sociedad del conocimiento" y "replantearía la educación para la ciudadanía en un mundo diverso e interconectado". Este documento, estaría mucho más orientado al segundo de los elementos, el de la educación virtual como nuevo paradigma, por ello lo desarrollaremos más delante.

Los resultados de las pruebas del LLECE y PISA van desplazando el foco de la evaluación de los aprendizajes al de la evaluación de los docentes. Se va construyendo un imaginario que la educación tiene serios problemas de calidad y que los educadores tienen un gran peso en esta negativa realidad. Se comienza a plantear la necesidad ahora de evaluar a los docentes, algo que ya se venía haciendo en Chile y que extiende por todo el continente (México, Colombia, entre otros).

Resulta especialmente aleccionador el caso de México, en el cual la reforma del ex presidente Peña Nieto, intento desarrollar evaluaciones punitivas a los docentes a través del Instituto Nacional de Evaluación de la Educación (INEE). Algo que le resultó imposible de alcanzar por la resistencia de la Coordinadora Nacional de Trabajadores de la Educación (CNTE) de ese país. 
Y en esa carrera llegamos a la crisis educativa, de perspectiva educativa, asociada a la pandemia del Coronavirus. La forma como se ha generado la suspensión de actividades presenciales en muchos países y la desorientación que ello ha causado no solo en el gremio educativo, sino en la población en general, ha contribuido a fortalecer la cultura evaluativa, en este caso sobre la capacidad de los docentes de prever y proveer que se presentan en el Informe Faure (UNESCOFaure, 1973 / Faure y Otros, 1973).

La nueva máquina educativa de la tercera revolución industrial se abre paso con el paradigma de la educación virtual. La nueva máquina no es la educación virtual, sino que esta sirve para terminar de desajustar e iniciar la desinstalación de la máquina educativa newtoniana.

\section{Educación virtual como el nuevo paradigma}

Entre 1961 cuando se conoce Unimate y en el cual aparece la tercera generación de computadoras con la IBM 360, hasta la presentación en 1977 de la computadora personal Apple II, el epicentro de la innovación y el conocimiento se trasladan aceleradamente de eje.

La instalación de "Unimate", el primer robot industrial colocado en General Motors de Ewing permitiría vincular los campos de la informática y la robótica en los medios de producción. La computación de tercera generación y la robótica industrial posibilitaron el desarrollo de la tercera revolución industrial, al escalar la automatización y la incorporación de "mano de obra" no humana a los ciclos productivos.

En ese contexto es en el cual se generan el Informe Faure y los intentos por reducir la responsabilidad de los Estados nacionales, tema del cual ya hablamos en el anterior punto. Cuando Faure señala que "la sociedad rechaza los productos de la educación" está planteándose la inquietud sobre las pocas respuestas que las escuelas, liceos y universidades están dando respecto a lo nuevo, lo cual preocupa al capital. Los ministerios de educación responden con lentitud a las exigencias del mercado, pero no por ello dejan de desinvertir en la educación pública afectando con ello a los más pobres. 
Pareciera que para los sistemas escolares el desembarco de la tercera revolución industrial fue un tema de artefactos y equipos, de innovaciones externas a su quehacer, no percibiendo el impacto de lo nuevo en las demandas que comenzaban a girar en torno a las instituciones educativas y, sobre todo como la escuela-máquina newtoniana perdía sentido ante la nueva realidad.

Al venir la escuela de una velocidad moderada en la percepción cotidiana de la aceleración de la innovación científico tecnológica, se pensaba que todas estas "novedades" impactarían a lo educativo décadas después, cuando los gobiernos tuvieran dinero para comprar los equipos con los cuales enseñar en las escuelas.

Las escuelas acostumbradas a "preceder" no entendieron que ahora estaban haciendo arqueología conceptual y paradigmática, y que este rol estaba siendo disputado por la creciente influencia de la mass media y por la construcción de una cultura del consumo de nuevas tecnologías.

El papel del complejo industrial cultural adquirió una relevancia de primer orden en la construcción de imaginarios, narrativas y perspectivas, de apropiación de lo emergente, tareas antes asignadas a las escuelas. A pesar de ello, contadas universidades de formación docente (inicial y continua) y centros de formación de formadores, incorporaron en su pensum el estudio de las mercancías del cine, la televisión, la música, el comic, la prensa en la construcción de conocimiento.

Los contados estudios sobre las mercancías del complejo industrial cultural eran análisis críticos de su rol, pero no terminaban de entender la disputa cognitiva, la tensión que esto generaban respecto a las instituciones educativas. La televisión a color, el emerger de la industria del video clip en los ochenta reafirmaron esta tendencia.

La aparición de las computadoras portátiles Epson HX-20 (1981), Osborne 1 (1981) y Microtor I (1985) dejaron claro que la informática y la computación iba a estar pronto al alcance de todos. Las críticas se multiplicaban sobre la incapacidad de las escuelas para desarrollar lo nuevo, mientras los centros de formación docente lo veían como una externalidad que no llegaría a las escuelas antes de jubilarse los actores de ese momento. Los gremios docentes comenzaron a 
desarrollar un discurso anti tecnología justificado en la imposibilidad de acceso a ella por las desigualdades sociales existentes, algo que, si bien era cierto, impedía pensar la dimensión pedagógica de lo que se venía.

La consola Nintendo 64 puso en contacto a millones de niños y jóvenes con el mundo digital, con la informática vinculada a la cotidianidad. Aunque no fue la primera consola de vídeo juegos, al menos la más popular en América Latina y la que se popularizo en el lenguaje y ocasionalmente uso de los sectores populares.

Las escuelas vieron a los vídeo juegos como distracción respecto al tiempo de aprendizaje en casa y, la formación docente no enseñó la lógica funcional de estas dinámicas, ni exploró la posibilidad de su uso con fines educativos. Fueron contados los estudios en este sentido, pero con un impacto limitado en la formación docente y la práctica cotidiana de los educadores.

La llegada de la Internet en la década de los noventa, el paso del sistema operativo MS-DOS (1985) a Windows 95, la popularización de las páginas web, implicaron un quiebre de diálogo generacional entre los llamados "nativos digitales" y sus predecesores "los inmigrantes digitales", mucho más con los "analfabetos tecnológicos".

Muy pocos ministerios de educación y gremios docentes trabajaron el impacto de los tecnológico en lo educativo más allá de la dotación como problema, parecieran que la ola de choque nunca iba a llegar o estaban a la espera de un modelo para copiar.

Precisamente las Organizaciones No Gubernamentales (ONGs) internacionales y las corporaciones informáticas como Google o Microsoft vieron este hueco en el tablero y concentraron una parte importante de sus presupuestos al desarrollo de una nube educativa y miles de contenidos digitales asociados, así como programas de alfabetización digital; la neo privatización educativa estaba en marcha, ahora soportada por la disputa del espacio y contenidos digitales. Del otro lado, desde las resistencias anticapitalistas los esfuerzos por presentar alternativas para otro uso del mundo digital en el aula, en armonía con propuestas emancipadoras, eran prácticamente inexistente. 
Las redes sociales, y su uso masivo, ya no solo por los jóvenes sino directamente por un $20 \%$ e indirectamente por un $80 \%$ de la población mundial, mostraba que la hegemonía de la era digital era ya un hecho concreto. A pesar de las posibilidades de uso de algunas de ellas para la construcción de resistencias sociales como lo han demostrado los neo zapatistas, el movimiento somos 132, la juventud de Chile, el movimiento 15M, las feministas y más recientemente los chalecos amarillos, la izquierda pedagógica guardo distancia respecto al "virus" de la educación digital en el aula.

Cuando voy a dar una conferencia en cualquier país o auditorio donde están los docentes hago una rápida encuesta visual y encuentro que por lo menos el $90 \%$ de ellos cuentan con un celular habilitado para el trabajo con redes sociales, vídeos, interactividad etc. Pero, el problema es que no solo un importante grupo de ellos no saben explotar sus potencialidades, sino que muchos ni siquiera tienen la disposición a aprender, como si esto no les fuera a afectar.

A ello contribuye el trabajo gremial centrado en los económico, la estabilidad y carrera docente con un desdén por lo pedagógico alternativo. Ello se evidencia en las pocas publicaciones pedagógicas del movimiento magisterial anticapitalista o su bajo volumen de impresión, así como en las contadas páginas web gremiales dedicadas al debate pedagógico.

No existe cultura de la lectura digital de documentos y libros, pero tampoco se imprimen; el pragmatismo de las llamadas "capsulas de mensajes alternativos", pretendiendo emular la lógica de los más jóvenes, esconde que se ha descuidado en la formación crítica de las nuevas generaciones de dirigentes gremiales y sindicales. Por supuesto ello tiene excepciones con esfuerzos como los que realiza la Confederación de Trabajadores de la Educación de la República Argentina (CTERA), Federación Colombiana de Trabajadores de la Educación (FECODE) y ahora ASOPROF, sin embargo, en los últimos tiempos, con limitada cobertura.

En el año 1996, un conjunto de Rectores de universidades de América Latina y el Caribe, reunidos en la Habana, Cuba, generaron en la Declaración de la Habana (1996), para que se celebrará una Conferencia Mundial de Educación Superior (CMES). La Declaración en cuestión expresó la preocupación de las autoridades universitarias sobre el impacto de lo tecnológico en el derecho a la educación. La virtualización surgía como una preocupación. 
La convocatoria a la $C M E S$ plantea una ambiciosa agenda que contiene los siguientes puntos: 1 - Las exigencias del mundo del trabajo. 2 - La educación superior y el desarrollo sostenible. 3 La contribución al desarrollo nacional y regional. 4 - La formación del personal de la educación superior: una misión permanente. 5 - La educación superior para una nueva sociedad: la visión estudiantil. 6 - De lo tradicional a lo virtual: las nuevas tecnologías de la información. 7 - La educación superior y la investigación: desafíos y oportunidades. 8 - La contribución de la educación superior al sistema educativo en su conjunto. 9 - Mujeres y educación superior: cuestiones y perspectivas. 10 - Promover una cultura de paz. 11 - Movilizar el poder de la cultura. 12 - Autonomía, responsabilidades sociales y libertad académica.

El debate sobre virtualidad, mundo digital y aceleración de la innovación no fueron concluyentes y se mantuvieron en las Conferencias de Cartagena (2008) y la Conferencia de Córdoba (2018). Lo que quedaba claro, era la distancia entre lo que discutíamos los universitarios y la demanda del entorno de la tercera revolución industrial y el curso hacia una cuarta revolución industrial.

La academia continuó siendo el refugio más importante para defender los grandes valores de la humanidad, la defensa de los derechos sociales entre ellos la educación y para la democratización del saber, pero ciertamente expresa hoy un déficit de actualización paradigmática.

En esa ruta UNESCO publicó dos textos que expresaban la presión del mercado y el modo de producción capitalista por el uso de la tecnología de punta en la educación. El primero de ellos, denominado Directrices para las políticas de aprendizaje móvil (UNESCO, 2013a), y el segundo, El futuro del aprendizaje móvil: implicaciones para la planificación y formulación de políticas (UNESCO, 2013a). Ambos documentos los analizaremos más en detalle más adelante, pero en este momento nos interesa destacarlos como antecedentes de la elaboración del UNESCO Science report: Towards 2030, donde se hace un inventario detallado de las capacidades institucionales existentes en el mundo en materia de investigación, arquitectura tecnológica e innovación.

Ese mismo año, se realiza en China una reunión para trabajar la convergencia del desarrollo científico, tecnológico y digital con el ODS4 recién aprobado por la ONU. De esa reunión 
emana la llamada Declaración de Qingdao: Dieciséis oportunidades digitales, transformación de la educación (UNESCO, 2015b). Los asistentes elaboran una Declaración, en cuyo numeral 3 de su preámbulo plantean que:

Inspirados por una visión humana de la educación basada en los derechos humanos y la justicia social, afirmamos que hay avances notables en TIC, y la expansión de la conectividad a Internet ha hecho que el mundo de hoy esté cada vez más interconectado y entregado al conocimiento y la familiaridad con las TIC, lo que es esencial para todos los hombres y mujeres.

En esta Declaración de Quingdao, continúan expresándose en el numeral 4 señalando que "para lograr los objetivos de inclusión y equidad de la calidad de educación y de los aprendizajes para toda la vida como como meta 2030, las TIC - Incluyendo el aprendizaje móvil - deben ser aprovechadas para reforzar la educación”. Luego en su numeral siete introducen el concepto de "Recursos Educativos Abiertos (REA)” y soluciones abiertas, colocando la actualización en la externalidad de las escuelas, desestimando la capacidad endógena de desarrollo de habilidades docentes y de capacidades institucionales para enfrentar los desafíos de la aceleración de la innovación en la educación. Este texto, constituye un marco conceptual para la privatización de la educación vinculada al desarrollo de las tecnologías de la comunicación.

En mayo de 2019, la UNESCO convocó en Beijing, China, la Conferencia internacional el lema "Planificar la educación en la era de la IA: un paso más hacia adelante" (UNESCO, 2019), la cual tuvo como propósitos:

- Debatir sobre las posibilidades de anticipar las competencias necesarias para vivir en la era de la IA y compartir las experiencias sobre el desarrollo de estas competencias, algo de debe permitir que las personas se adapten a una sociedad en la que la IA tenga su lugar;

- Intercambiar sobre las tendencias más recientes de la IA y la manera en que ayudan a diseñar la educación y el aprendizaje; 
- Evaluar las lecciones sacadas a partir de las nuevas políticas y estrategias nacionales que permiten la utilización de la IA como instrumento para alcanzar el ODS 4; y

- Reforzar la cooperación y las alianzas internacionales con el objetivo de promover una utilización equitativa, inclusiva y transparente de la IA en la educación.

De este evento (UNESCO 2019) emanó el llamado "Primer consenso sobre la inteligencia artificial y la educación”. A diferencia de otros instrumentos emanados de conferencias mundiales, esta declaración es contundente y precisa al señalar la inminencia e impostergabilidad del cambio de $360^{\circ}$ en espiral ascendente y concéntrica, que los reunidos:

Revisamos las tendencias recientes en la evolución de la IA y su profundo impacto en las sociedades humanas, las economías y el mercado laboral, así como en la educación y los sistemas de aprendizaje permanente. Examinamos las implicaciones de la IA para el futuro del trabajo y el desarrollo de habilidades y consideramos su potencial para remodelar las bases fundamentales de la educación, la enseñanza y el aprendizaje.

Concluyéndose entonces que se debe tener "en cuenta la naturaleza multidisciplinaria de la IA y su impacto en los aprendizajes". Es decir, mientras los sistemas educativos en el mundo están en caos por el uso de la virtualidad, el sistema en su conjunto requiere del uso educativo de la inteligencia artificial para seguir avanzando.

\section{REFERENCIAS}

Conferencia de Córdoba. (2018). Conferencia Regional de Educación Superior de América Latina y el Caribe (Colección CRES). Resúmenes Ejecutivos. Córdoba: UNESCO. https://www.iesalc.unesco.org/2019/07/17/coleccion-cres-2018-conferencia-regional-deeducacion-superior-de-america-latina-y-el-caribe-cordoba-2018-resumenes-ejecutivos/. 
Conferencias de Cartagena. (2008). Declaración y Plan de Acción de la Conferencia Regional de Educación Superior en América Latina y el Caribe (CRES). Instituto Internacional de la UNESCO para la Educación Superior en América Latina y el Caribe (IESALC) y por el Ministerio de Educación Nacional de Colombia.

Declaración de La Habana. (1996). Conferencia Regional de la UNESCO sobre Políticas y Estrategias para la Transformación de la Educación Superior en América Latina y el Caribe. UNESCO / Centro Regional para la Educación Superior en América Latina y el Caribe (CRESALC) / Ministerio de Educación de Cuba. Del 18 al 22 de noviembre de 1996. Ha Haba: UNESDOC-UNESCO. https://unesdoc.unesco.org/ark:/48223/pf0000161857.locale=es/.

Delors, J. y Otros. (1996). La Educación Encierra un Tesoro. Comisión Internacional sobre la Educación para el Siglo XXI. Madrid: Editorial Santillana-UNESCO y UNESDOCUNESCO. https://unesdoc.unesco.org/ark:/48223/pf0000109590_spa/.

El Confidencial. (2018). "El revelador libro del gurú de PISA sobre el éxito educativo (y qué dice sobre España)”. España: Andreas Schleicher. https://www.elconfidencial.com/alma-corazon-vida/2018-06-14/andreas-schleicher-pisaexito-educativo-espana_1578377/.

LLECE-UNESCO. (2019). Estudio Regional Comparativo y Explicativo (ERCE). Laboratorio Latinoamericano de Evaluación de la Calidad Educativa (LLECE). Santiago: https://es.unesco.org/fieldoffice/santiago/llece/ERCE2019/.

LLECE-UNESCO. (2013). Tercer Estudio Regional Comparativo y Explicativo. Análisis Curricular. Laboratorio Latinoamericano de Evaluación de la Calidad Educativa (LLECE). Santiago: Publicado por la Oficina Regional de Educación para América $\begin{array}{llll}\text { Latina } & \mathrm{y} & \text { el }\end{array}$ https://unesdoc.unesco.org/ark:/48223/pf0000227501/. 
LLECE-UNESCO. (2004-2008). Segundo Estudio Regional Comparativo y Explicativo. Los aprendizajes de los estudiantes de América Latina y el Caribe. Laboratorio Latinoamericano de Evaluación de la Calidad Educativa (LLECE). Santiago: UNESDOC-UNESCO. https://unesdoc.unesco.org/ark:/48223/pf0000160659/.

LLECE-UNESCO. (1997). Primer Estudio Regional Comparativo y Explicativo. Evaluación de la Calidad de la Educación en América Latina. Laboratorio Latinoamericano de Evaluación de la Calidad Educativa (LLECE). México: UNESCO. https://es.unesco.org/fieldoffice/santiago/llece/PERCE1997/.

UNESCO. (2019). Conferencia Internacional sobre Inteligencia Artificial en la Educación en Beijing. De 16-18 de mayo de 2019. Beijing: UNESCO.

UNESCO. (2016a). Declaración de Lima: Balances y Desafíos para la Agenda Educativa Regional 2015-2030. 30 y 31 de agosto de 2014. Organización de las Naciones Unidas para la Educación, la Ciencia y la Cultura. Lima: UNESDOC Biblioteca Digital. https://unesdoc.unesco.org/ark:/48223/pf0000244335/.

UNESCO. (2016b). Educación 2030 Declaración de Incheon. Hacia una educación inclusiva y equitativa de calidad y un aprendizaje a lo largo de la vida para todos. http://www.unesco.org/new/fileadmin/MULTIMEDIA/FIELD/Santiago/pdf/ESP-Marcode-Accion-E2030-aprobado.pdf/.

UNESCO. (2015a). Replantear la educación ¿Hacia Un bien común mundial? Paris: Ediciones UNESCO. http://eduteka.icesi.edu.co/pdfdir/unesco-replantear-educacion.pdf/.

UNESCO. (2015b). Declaración de Qingdao (2015). Qingdao: UNESCO. https://unesdoc.unesco.org/ark:/48223/pf0000233352/.

UNESCO. (2014). Educación para Todos (EPT) en América Latina y el Caribe: Balance y Desafíos post-2015. Declaración de Lima. De 30 - 31 de octubre del 2014. Lima: UNESDOC-UNESCO. https://unesdoc.unesco.org/ark:/48223/pf0000230628/. 
UNESCO. (2013a). Directrices de la UNESCO para las políticas de aprendizaje móvil. Paris: UNESCO. https://unesdoc.unesco.org/ark:/48223/pf0000219662/.

UNESCO. (2013b). El futuro del aprendizaje móvil. Implicaciones para la planificación y la formulación de políticas. Paris: UNESCO. http://www.aprendevirtual.org/centrodocumentacion-pdf/Aprendizaje\%20movil\%20UNESCO.pdf/.

UNESCO. (2011). Décima Reunión del Grupo de Alto Nivel sobre Educación para Todos (EPT). Discurso de la Directora General de la UNESCO Irina Bokova. 22 de marzo de 2011. Jomtien: Servicio de Prensa de la UNESCO. http://www.unesco.org/new/es/media-services/singleview/news/high_level_group_2011_director_general_calls_for_restored_u/.

UNESCO. (2000). Marco de Acción de Dakar. Educación para Todos: cumplir nuestros compromisos comunes. Adoptado en el Foro Mundial sobre la Educación Dakar (Senegal), 26-28 de abril de 2000. Paris: UNESDOC-UNESCO. https://unesdoc.unesco.org/ark:/48223/pf0000121147_spa/.

UNESCO-EPT. (2015). Educando en igualdad. Educación para Todos. UNESCO. http://www.educandoenigualdad.com/wpcontent/uploads/2015/05/EPT2015.compressed.pdf/.

UNESCO-Jomtien. (1990). Conferencia Mundial sobre Educación para Todos: Satisfacción de las Necesidades Básicas de Aprendizaje. Mayo de 1990. New York: UNESDOCUNESCO. https://unesdoc.unesco.org/ark:/48223/pf0000127583_spa/.

UNESCO-Faure. (1973). Informe Faure / Faure, E. y Otros (1973). Aprender a ser. La educación del futuro. Madrid: Alianza Editorial-UNESCO. https://www.berrigasteiz.com/monografikoak/inklusibitatea/pubs/unesco_aprender\%20a \%20ser.pdf/. 